\title{
Vacancy Cluster: Helium Synergy in Void Nucleation
}

\author{
KENNETH C. RUSSELL \\ Vacancy clusters produced in displacement cascades may capture helium to give void nucle- \\ ation. Rate equations are derived for such nucleation. Most of the helium is captured and \\ sequestered early on in most irradiations by a low number density of small voids. This capture \\ may terminate void nucleation early in irradiation, in agreement with many experimental \\ studies.
}

DOI: $10.1007 / \mathrm{s} 11661-007-9352-\mathrm{x}$

(C) The Minerals, Metals \& Materials Society and ASM International 2007

\section{INTRODUCTION}

RADIATION-INDUCED void swelling was first observed in the 1960s during materials development for fuel cladding and ducts for the fast breeder reactor. ${ }^{[1,2]}$ High number densities of nanometer-micrometer diameter helium-containing voids (or cavities) were observed. The resulting volumetric swelling percentages were unacceptable in the reactor. There was a large international effort to understand the physical basis of the swelling and thereby to eliminate or at least control it.

Theories for void nucleation from simultaneous supersaturations of vacancies and self-interstitials were derived simultaneously by Russell ${ }^{[3]}$ and Katz and Wiedersich. ${ }^{[4]}$ The resulting steady-state nucleation equations were much the same. Self-interstitial involvement was found to give a large, up to order of magnitude increase in the critical nucleus size. Self-interstitial involvement was found to reduce the nucleation rate by a few orders of magnitude, which is a relatively modest factor in the context of nucleation theory.

Neutron irradiation produces helium through $(n, \alpha)$ transmutation reactions. Concentrations typically reach the parts per million (atomic) range in breeder reactor materials and may be several orders of magnitude higher under the high-energy neutron regimes of proposed thermonuclear reactors. Helium may also be introduced into the lattice by $\alpha$-particle irradiation from an ion accelerator. Numerous experiments had shown that helium may give a major increase in void nucleation kinetics and the ensuing swelling rate.

Prior to void formation, most helium atoms are trapped in vacant lattice sites. However, reaction with an impinging self-interstitial is energetically favorable and puts the helium atom into an interstitial site ${ }^{[5]}$ where it can rapidly diffuse.

KENNETH C. RUSSELL, Professor Emeritus of Metallurgy and Nuclear Engineering, is with the Departments of Materials Science and Engineering and Nuclear Science and Engineering, Massachusetts Institute of Technology, Cambridge, MA, USA. Contact e-mail: kenruss@mit.edu

This article is based on a presentation given in the symposium entitled "Solid-State Nucleation and Critical Nuclei during First Order Diffusional Phase Transformations," which occurred October 15-19, 2006 during the MS\&T meeting in Cincinnati, Ohio under the auspices of the TMS/ASMI Phase Transformations Committee.

Article published online November 1, 2007
Theories for the effect of helium on void nucleation were independently developed by Wiedersich et al..$^{[6-9]}$ and by Russell et al. ${ }^{[10-18]}$ Two different approaches were taken to void nucleation in the presence of mobile helium. In the first, ${ }^{[7,8,12]}$ difference equations were written to describe the movement of clusters in a phase space of void size and helium content and were numerically solved. Helium was predicted to significantly promote void nucleation under some conditions, in rough agreement with experiments.

Unfortunately, the difference equations are slow to converge and tend to be unstable. In addition, the calculation is physically opaque, which makes it hard to do a sensitivity analysis to determine the role of different variables. The opacity may also allow serious errors to go undetected.

The other approach is to consider individual mechanisms of void formation. Katz and Wiedersich ${ }^{[6,9]}$ and Parker and Russell ${ }^{[17,18]}$ calculated heterogeneous void nucleation rates on vacancy:helium embryos. Parker and Russell also calculated the number densities of the various size vacancy:helium embryos. The nucleation rates on the various sizes of embryos were calculated and added to give the overall nucleation rate.

Major reactor incidents at Three Mile Island (1979) in the United States and Chernobyl (1986) in the Ukraine caused a sharp drop in interest in nuclear electricity and associated radiation effects. Today there is a renewed interest in nuclear electricity.

In addition, several developments have advanced the understanding of the irradiated state. Recent molecular dynamics studies of displacement cascades ${ }^{[19-22]}$ have shown that in less than about a nanosecond about one-fourth of the interstitials are incorporated into stable clusters. These interstitials never become part of the point defect sea. The resulting "production bias"[23] leaves a very appreciable excess of vacancies to nucleate voids. Elastic interaction between dislocations and selfinterstitials is not needed to give the vacancy excess leading to void nucleation and growth.

Field ion microscopy ${ }^{[24]}$ and recent Monte Carlo calculations $^{[25-27]}$ have shown that a similar level of vacancy clustering in cascades occurs on the microsecond-millisecond time scale. Unlike the interstitial clusters, the vacancy clusters mostly decay to single, mobile vacancies that become part of the point defect sea. 
Russell and $\mathrm{Kim}^{[28]}$ have shown that these clusters may serve as embryos for critical void nuclei. The void nucleation rate may be greatly increased over what it would be in the absence of vacancy clustering.

This article analyzes the synergistic effects of vacancy clusters and mobile helium atoms on void nucleation. The analysis is based on the nodal line-critical point formalism and incubation time calculations. The analysis provides an understanding for helium effects in void nucleation, both with and without vacancy clustering in cascades. In addition, it supplies a simple scenario for the cessation of void nucleation early in the radiation process.

\section{POINT DEFECTS IN METALS}

Vacancies mostly exist as isolated vacant lattice sites. Self-interstitials are known to exist as dumbbells whereby two atoms share a single lattice site. The dumbbell may be split in $\langle 100\rangle,\langle 110\rangle$, or $\langle 111\rangle$ directions, depending on the material. ${ }^{[29]}$ The defect moves by exchanging one end of the dumbbell for a lattice atom on each jump. In a pure metal, an atom that is part of a split interstitial will move at most a few lattice spacings before being left behind. However, the split interstitial preferentially incorporates undersized lattice atoms that remain part of the dumbbell as it moves through the lattice. This migration is somewhat similar to movement of a vacancy:solute complex.

A solvent atom in a self-interstitial will spontaneously exchange places with a substitutional helium atom so that the helium becomes part of the split interstitial described earlier. ${ }^{[5]}$ The helium-containing dumbbell is highly mobile and may freely migrate.

Vacancies, self-interstitials, and helium interstitials are mobile at most temperatures of interest and migrate around the lattice until they encounter a sink, which may be a dislocation line, a grain boundary, or a void. Rigorous analyses of point defect behavior in irradiated metals are presented elsewhere. ${ }^{[30,31]}$ Some simple, useful rearrangements are presented here.

For many conditions of interest, dislocations are the main point defect sinks and thermal creation of vacancies is unimportant. Then, at steady state,

$$
\begin{gathered}
D_{i} C_{i}=K_{i} / Z_{i} \rho_{d} \\
D_{v} C_{v}=K_{v} / Z_{v} \rho_{d}
\end{gathered}
$$

where $i$ and $v$ refer to interstitials and vacancies, respectively, and $D=$ diffusivity, $C=$ concentration in defects per lattice atom, $K=$ defect production rate per lattice atom per second, $\rho_{d}=$ dislocation density in length per unit volume, and $Z_{i}, Z_{v}=$ dislocation sink strength constants, not to be confused with the Zeldovich factor.

The production rate of mobile vacancies is about onefourth greater than that of mobile interstitials because of the production bias discussed earlier.
In the case of helium $(x)$ trapped at dislocations,

$$
D_{x} C_{x}=K_{x}^{T} C_{T} / Z_{x} \rho_{d}
$$

where $K_{x}^{T}=$ rate of detrapping; $C_{T}=$ concentration of trapped helium, which is very close to the total concentraion; and $Z_{x}=$ dislocation sink strength constant for helium.

It is convenient to define the dislocation sink strength as $k_{d}^{2}=Z \rho_{d}$, where $Z$ refers to vacancies, self-interstitials, or mobile helium atoms, as the case may be. One may expect that the sink strength constants are not far from unity with $Z_{i}$ perhaps 10 pct greater than $Z_{v}$ because of elastic interactions.

Detrapping of helium lying on a dislocation line is fairly difficult. An approaching self-interstitial is likely to simply annihilate at the dislocation line without affecting the trapped helium. Even if the helium is detrapped, it is very likely to be retrapped in a very few jumps.

However, the dislocations are not stationary but are rapidly climbing because of the excess of arriving vacancies over self-interstitials. The dislocation line will climb by a lattice parameter in roughly the time it takes a self-interstitial to react with a trapped helium. Thus, the helium atoms will be left behind in lattice sites where easy detrapping may occur.

It is useful to introduce the quantities

$$
\beta^{o}=D C / a^{2}
$$

for the arrival rates of the various defects at a sink in a single atomic volume in size; $a=$ lattice spacing. Then, for substitutional helium, $K_{x}^{T}=\beta_{i}^{o}$ and

$$
\beta_{x}^{o}=\beta_{i}^{o} C_{T} /\left(Z_{x} \rho_{d} a^{2}\right)
$$

In the common breeder reactor example of $\rho_{d}=$ $10^{10} / \mathrm{cm}^{2}$ and $C_{T}=10^{-6}, \beta_{x}^{o} \sim \beta_{i}^{o} / 10$ The similarity of the two arrival rates is a bit surprising, considering the low concentration of helium. In proposed thermonuclear reactors, the first wall is subject to $14.1 \mathrm{MeV}$ neutron bombardment, which gives high helium formation rates. It is, thus, possible that $\beta_{x}^{o} \gg \beta_{i}^{o}$. The direction of the inequality is surprising, because earlier thinking had the helium arrival rate at voids much lower than that of selfinterstitials and vacancies.

Voids are excellent sinks for helium atoms and constitute what might be called deep traps in that detrapping is much more difficult than from vacancies. A helium atom will be detrapped only if struck hard enough to drive it back into the lattice. The required energy will be in the $10 \mathrm{~s}$ of electron volts $(\mathrm{eV})$ range, similar to that for atomic displacements in the lattice. We may then roughly equate the detrapping rate to the atomic displacement rate. This rate may be orders of magnitude lower than the detrapping rate from vacancies.

The sink strength of voids of radius $r_{c}$ and number density $\rho_{c}$ is given by $k_{c}^{2}=4 \pi \rho_{c} r_{c}$. If the helium is trapped only by voids,

$$
\beta_{x}^{o}=K_{x}^{c} C_{T} /\left(4 \pi a^{2} r_{c} \rho_{c}\right)
$$


The concentration of mobile helium will be decreased by about half when the helium is equally distributed between shallow substitutional traps and deep void traps. The ratio of sink strengths for such equipartition may be calculated, requiring that the helium in each trap must be in equilibrium with the same concentration of mobile helium. A bit of algebra shows that the crossover occurs when

$$
K_{x}^{c} / \beta_{i}^{o}=k_{c}^{2} / k_{d}^{2}
$$

or

$$
K_{x}^{c} / \beta_{i}^{\mathrm{o}}=4 \pi \mathrm{r}_{c} \rho_{c} / \mathrm{Z}_{x} \rho_{d}
$$

As an illustration, take $\rho_{d}=10^{10} / \mathrm{cm}^{2}$ and, for small voids, $r_{c}=10^{-7} \mathrm{~cm}$. Then, if the detrapping rates $K_{x}^{c}$ and $\beta_{i}^{o}$ are the same, the trapped helium will be equally distributed between voids and dislocations for a void number density of $10^{16} / \mathrm{cm}^{3}$. In fact, the detrapping rates will probably differ by orders of magnitude so that a relatively small number density of voids will provide the dominant traps. Further void nucleation and growth will give a precipitous decrease in $\beta_{x}^{o}$ so that helium can no longer assist in void nucleation.

Small clusters of vacancies constitute medium depth traps. Take, for example, a cluster of three vacancies and one or more helium atoms. The capture of three self-interstitials in succession will restore the lattice and inject the helium into interstitial sites. The capture of four self-interstitials and one vacancy has the same effect. Vacancies and self-interstitials arrive at clusters at nearly the same rate, so that for a trimer, the detrapping rate, $K_{x}^{T}$, is within a factor of 10 of $\beta_{i}^{o}$. The detrapping rate will approach the value for large voids at cluster sizes of perhaps a dozen vacancies.

\section{POINCARÉ ANALYSIS}

Here, we analyze void nucleation using the nodal line, critical point analysis presented by Russell ${ }^{[15]}$ based on the analysis of Poincaré ${ }^{[32]}$ for celestial mechanics. The analysis follows the motion of a point in a phase space wherein the velocity depends only on the coordinates and not explicitly on the time. These are called "autonomous" or "stationary" equations. The velocity of the embryonic void is determined by the capture and loss rates of defects that depend only on the position in $(n, x)$ phase space. The Poincaré formalism is, therefore, applicable.

Figure 1 shows the various processes giving rise to void movement. The velocity $\dot{n}$ equals the capture rate of vacancies minus the capture rate of self-interstitials and the loss rate of vacancies. The velocity $\dot{x}$ is the capture rate of mobile helium minus the loss rate. Helium may be lost from the vacancy clusters by collision with an energetic lattice atom that drives it back into the matrix and by thermal emission.

$$
\begin{gathered}
\dot{n}=\beta_{v}^{o} n^{1 / 3}-\alpha_{v}-\beta_{i}^{o} n^{1 / 3} \\
\dot{x}=\beta_{x}^{o} n^{1 / 3}-\alpha_{x}-x K_{x}^{c}
\end{gathered}
$$

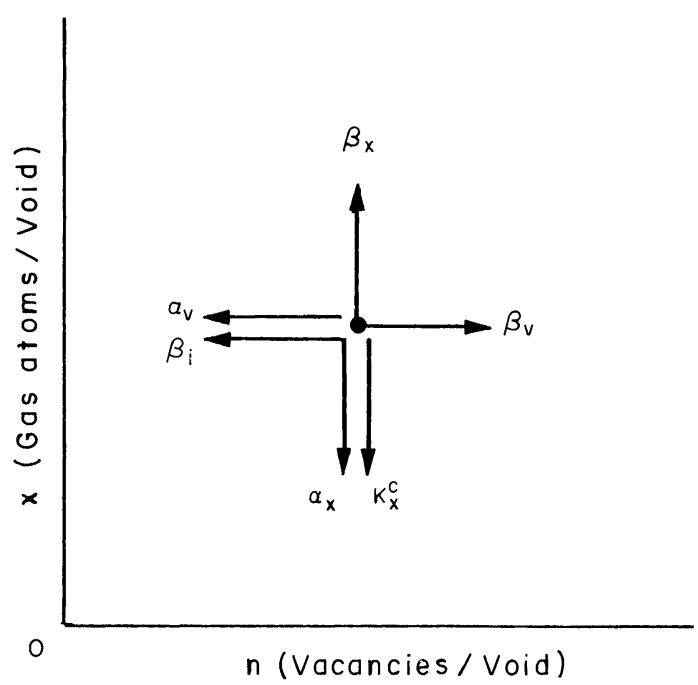

Fig. 1-Atomic processes involved in void nucleation showing mechanisms for vacancy and gas atoms capture and loss. Symbols are defined in the Nomenclature.

The defect emission rates $\alpha_{v}$ and $\alpha_{x}$ are obtained by applying detailed balancing to $\Delta G^{o}(n, x)$, the free energy of void formation in the absence of self-interstitial involvement.

The locus points for which $\dot{n}$ or $\dot{x}$ are zero is known as a nodal line. An intersection of the two nodal lines is known as a critical point. A cluster at a critical point is immobilized, neither gaining nor losing gas atoms nor vacancies. The $\dot{n}$ nodal line may only be crossed by clusters moving in the $x$ direction and the $\dot{x}$ nodal line by clusters moving in the $n$ direction.

In the case at hand, the nodal lines may take one of two configurations, depending on the material parameter $\Psi$, where

$$
\Psi=\left(9 \beta_{x}^{o} \ln S_{e} /\left(A^{2} K_{x}^{c}\right)\right.
$$

The effective supersaturation is

$$
S_{e}=\left(C_{v} / C_{v e}\right)\left(1-\beta_{i}^{o} / \beta_{v}^{o}\right)
$$

where $C_{v} / C_{v e}$ is the ratio of the actual and equilibrium vacancy concentrations.

The energetic parameter $A$ is

$$
A=\left(36 \pi \Omega^{2}\right)^{1 / 3} \gamma / \mathrm{kT}
$$

where $\Omega=$ atomic volume of solid, $\gamma=$ void: matrix surface energy, and $\mathrm{kT}=$ Boltzmann factor. The key variables in $\Psi$ are the effective supersaturation and the ratio of helium capture and detrapping rates.

At low vacancy supersaturations and helium arrival rates $\Psi<1$ and the nodal lines will intersect twice, as shown in Figure 2. The critical point at larger $n$ is known as a saddle, but is of no physical significance. The critical point at $\hat{n}, \hat{x}$, is known as a stable node. It attracts clusters from the regions around it, as shown by the arrows in the figure. Thus, the theory predicts that irradiation conditions giving nodal line intersection may lead to high number densities of very small helium-containing vacancy clusters that are trapped at the stable node. 


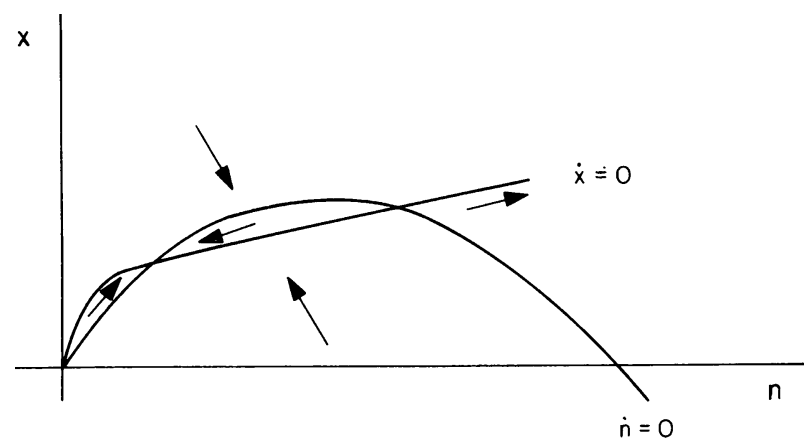

Fig. 2 -Schematic diagram of nodal lines for $\Psi<1$. Nearby clusters are attracted to the table node.

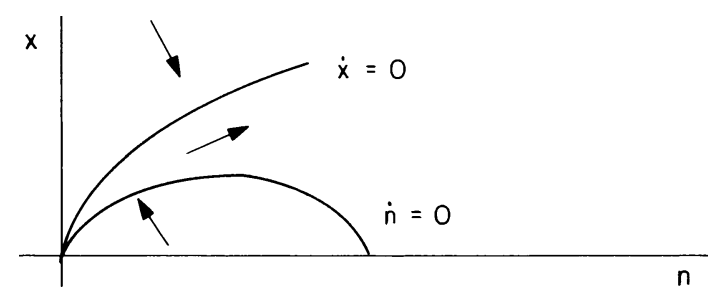

Fig. 3-Schematic diagram of nodal lines for $\Psi>1$. Voids below the $\dot{n}$ nodal loop lose vacancies and gain helium until reaching the nodal line. Voids between the nodal lines may grow by simultaneous capture of vacancies and helium.

Figure 3 corresponds to high vacancy supersaturations and high helium arrival rates. $\Psi>1$ and the nodal lines do not cross. Clusters beneath the $\dot{n}$ loop lose vacancies while capturing gas atoms until they cross the nodal line. Clusters to the left of the $\dot{x}$ nodal line capture vacancies and lose gas atoms until they cross the nodal line. Clusters between the nodal lines gain gas and vacancies simultaneously until reaching the peak of the $\dot{n}$ nodal loop. At this point, the clusters are nucleated and may grow by capturing vacancies and helium in any proportions.

The Poincaré analysis has a large advantage over the holistic numerical solutions of the myriad difference equations describing the various transitions in $(n, x)$ space ${ }^{[7,12]}$ in enabling the determination of the path of activation for void nucleation. Then only clusters involved in the path of activation need to be considered, which usually makes calculation of the nucleation rate relatively straightforward.

The Poincaré analysis gives only the average behavior of clusters. The fluctuations that give rise to nucleation must also be considered. The result is a hybrid treatment in which fluctuational nucleation theory is melded with the predictions of the Poincare analysis. The combination of the two analyses is explored in the ensuing sections of this article.

\section{NUCLEATION}

This section only outlines the equations for void nucleation. Detailed expressions are presented elsewhere. ${ }^{[3,17,18]}$
As just noted, when $\Psi>1$ stable voids may form by simultaneous capture of vacancies and helium without surmounting an activation barrier, or "spontaneous nucleation.'

$\Psi<1$ clusters up to the node at $(\hat{n}, \hat{x})$ form readily and may act as heterogeneous nucleation sites, as shown schematically in Figure 4. Figure 5 shows the progressive decrease in activation barrier with larger clusters.

Nucleation at constant helium content may occur if the time between helium arrivals is large compared with the time needed for nucleation by vacancy capture. We may then consider size fluctuations that take embryonic

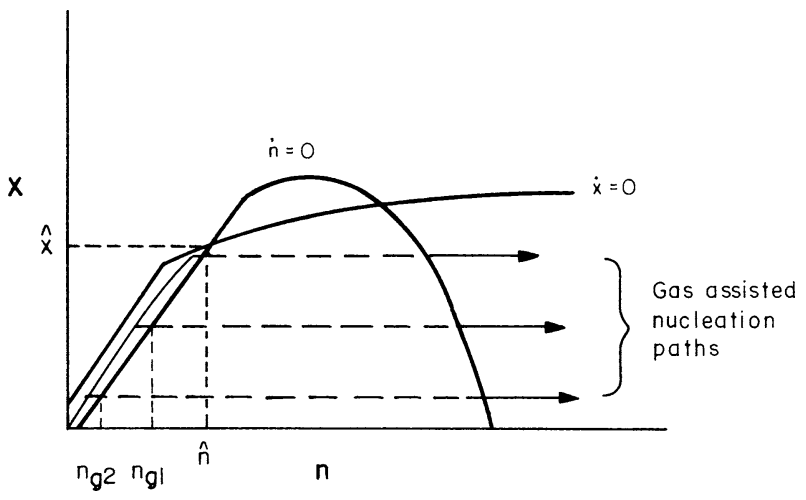

Fig. 4 -Nodal line configuration with $\Psi<1$. Clusters up to the size $(\hat{n}, \hat{x})$ may serve as heterogeneous nucleation sites. ${ }^{[18]}$

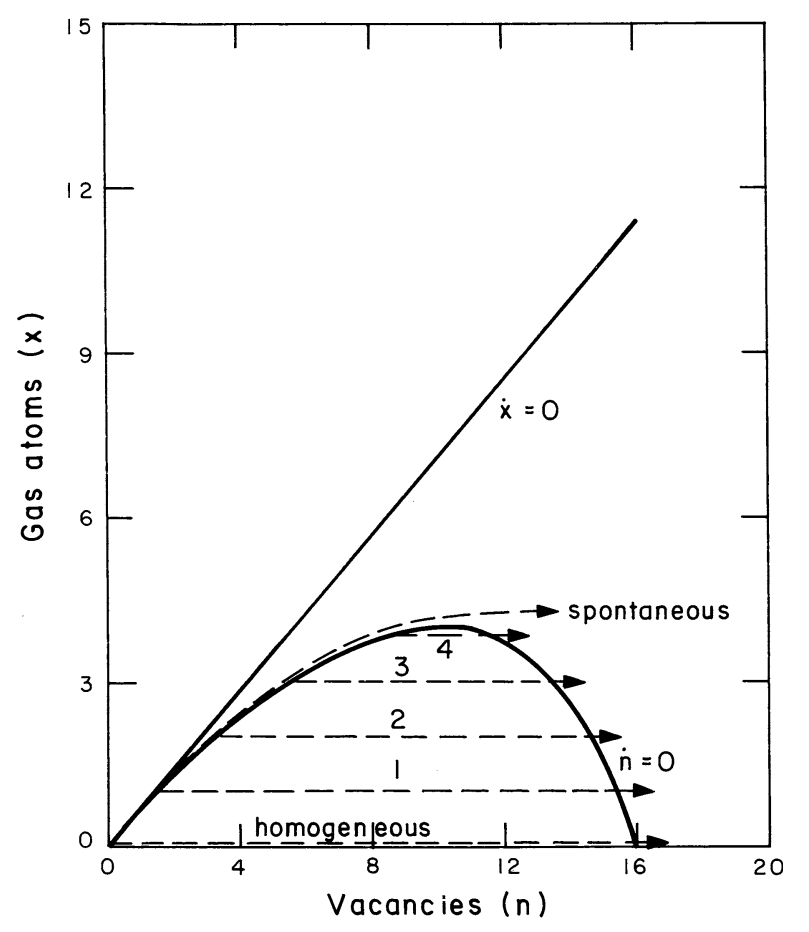

Fig. 5-Calculated void nucleation paths corresponding to simultaneous heavy ion and $\alpha$-particle irradiation of type 304 stainless steel. ${ }^{[18]}$ 
voids from the left side of the $\dot{n}$ nodal loop to the right, where they are nucleated.

The key quantity of gas-assisted void nucleation is $\Delta G^{\prime}(n, x)$, the radiation-modified activation barrier. This quantity is given by

$$
\Delta G^{\prime}(n, x)=\mathrm{kT} \sum_{j=n_{o}}^{j=n} \ln \left\{\left\lfloor\frac{\beta_{i}^{o}}{\beta_{v}^{o}}\right\rfloor+\exp \left(\frac{1}{\mathrm{kT}} \frac{\partial \Delta G^{o}(j, x)}{\partial j}\right)\right\}
$$

where $\Delta G^{o}(n, x)$ is the purely thermodynamic barrier to void nucleation, which includes contributions from vacancy supersaturation, surface energy, and gas pressure.

Setting $\frac{\partial \Delta G^{\prime}(n, x)}{\partial n}=0$ gives two extrema. The maximum is denoted $n^{*}$, the critical nucleus size. The minimum is denoted $n_{o}$, at which point the internal gas pressure so reduces vacancy emission that the void cannot shrink further.

The left side of the $\dot{n}$ nodal loop gives $n_{o}$, and the right side gives $n^{*}$. The activation barrier, $\Delta G^{*}$, is obtained by summation from $n=n_{o}$ to $n=n^{*}$. The steady-state nucleation rate on a number density $\rho(x)$ of clusters of $x$ gas atoms is

$$
J_{s}^{*}=Z \beta^{*} \rho(x) \exp \left(-\Delta G^{*} / \mathrm{kT}\right)
$$

The frequency factor, $\beta^{*}=\beta_{v}^{o} n^{* 1 / 3}$. The Zeldovich factor $Z=1 / \delta$, where $\delta=$ width of $\Delta G^{\prime}$, and $\mathrm{kT}$ is below the maximum at $\Delta G^{*} .^{[33]}$ Figure 6 shows $\Delta G^{\prime}$ for homogeneous nucleation and for nucleation on clusters of two and four vacancies.

Steady-state conditions will lag the onset of a supersaturation by an interval often termed the incubation time, here denoted $\tilde{\tau}_{\delta}$. Feder et al. ${ }^{[33]}$ give a detailed analysis of incubation times in the context of condensation of liquid droplets from the vapor, but their results apply to nucleation processes in general. Their analysis is based on the principle of time reversal symmetry, which requires that fluctuations form and decay by the same path. Feder et al. considered the behavior of a critical nucleus, which is unstable and will either grow to macroscopic size or decay to single atoms. It is easy to calculate the path and rate of decay, which is then equated to the time and path for fluctuation formation.

Feder et al. showed that the critical nucleus moved by random walk on the essentially flat portion of the activation barrier until it became subcritical by an amount, kT. The particle then steadily decayed to monomer. They found that the longest time $(\sim 75$ pct of the total) was spent in random walk. The incubation time was then

$$
\tau_{\delta}=\delta^{2} / 2 \beta^{*}
$$

or

$$
\tau_{\delta}=\left(2 Z^{2} \beta^{*}\right)^{-1}
$$

The incubation time depends on the shape of the activation barrier rather than the height. A broad barrier will favor a large $\tau_{\delta}$, regardless of height. Conversely, the steady-state nucleation rate only weakly depends on the shape of the barrier and strongly on the height.

\section{CHARACTERISTIC TIMES}

The results of these calculations depend on certain characteristic times. We define $\tau_{o}=1 / \beta^{o}$. The quantity $t_{v}^{o}, \tau_{i}^{o}$, and $\tau_{x}^{o}$ are then the times between arrivals of vacancies, self-interstitials, or helium atoms at a sink of a single vacancy.

The arrival rate at an $n$-mer (cluster of $n$ vacancies) varies as $n^{1 / 3}$. The critical nuclei of interest are likely to contain at most a few $10 \mathrm{~s}$ of vacancies so that the difference between arrival times at a single vacancy and a critical nucleus is a factor of about 2 or 3 . Such a factor is unimportant in the order of magnitude estimates to follow.

Subcritical size vacancy embryos may be formed in the displacement cascades or by statistical fluctuations. By the preceding reasoning, these clusters will have a lifetime of about $\tau_{\delta}$, during which they may decay to single vacancies, achieve critical nucleation size, or capture one or more helium atoms. With critical nuclei of $10 \mathrm{~s}$ of vacancies, $\tau_{\delta}$ is between about $10 / \beta_{v}^{o}$ and $100 / \beta_{v}^{o}$.

Nucleation on a number density $\rho(x)$ of helium clusters will be largely complete after a time $\tau_{J}$ for which

$$
J_{s}^{*} \tau_{J} \sim \rho(x)
$$

Then

$$
\tau_{J}=\left[Z \beta^{*} \exp \left(-\Delta G^{*} / \mathrm{kT}\right)\right]^{-1}
$$

Strictly speaking, one should also consider the incubation time to establish steady state, but for any situation of interest, $\tau_{j}>>\tau_{\delta}$.

The final characteristic time is that for irradiation, $\tau_{r}$. This time will be the order of minutes or hours for charged particle radiations or of months or years for radiations in nuclear reactors.

Six characteristic times are of interest. Three are for arrival rates of point defects, one for embryo lifetime, one for nucleation time, and one for radiation time.

\section{PATH OF ACTIVATION WITHOUT CLUSTER INJECTION}

Consider first the case where the mobile helium arrival rate is so high that $\tau_{x}$ is the smallest of the six characteristic times. The inequality $\Psi>1$ will certainly be satisfied. Clusters will rapidly form between the $\dot{n}$ and $\dot{x}$ nodal lines to the $\dot{n}$ maximum, where they are nucleated.

Assume now that $\tau_{v}^{o}<\tau_{x}^{o}$ and $\Psi>1$. Clusters may still form by agglomeration of vacancies and helium atoms to move to the top of the $\dot{n}$ nodal loop, but may also act as embryos for nucleation. If $\tau_{x}<\tau_{j}$, the cluster 
will capture more helium to move up the $\dot{n}$ nodal line to where the activation barrier is smaller. If a point is reached where $\tau_{J}<\tau_{x}$, nucleation will occur. Otherwise, the clusters will reach the top of the $\dot{n}$ nodal line to give spontaneous nucleation.

If $\Psi<1$, the nodal lines cross, and the clusters may spontaneously capture gas only up the stable node at $(\hat{n}, \hat{x})$. These clusters will gain vacancies to develop into critical nuclei if the observation time is long enough, e.g., $\tau_{J}<\tau_{r}$.

The roles of these various paths of activation in void nucleation are illustrated by the modeling study of Parker and Russell ${ }^{[18]}$ for dual heavy ion and $\alpha$-particle irradiation of type 304 stainless steel. Figures 5 through 7 show the results of calculations for $873 \mathrm{~K}$ at a displacement rate of $2.3 \times 10^{-4} \mathrm{dpa} / \mathrm{s}$ and a helium injection rate of $3.0 \times 10^{-2} \mathrm{appm} / \mathrm{s}$. Figures 5 and 6 correspond to $5000 \mathrm{~s}$, where the helium concentration is 150 appm (atomic parts per million).

Figure 5 shows the possible nucleation paths. The parameter $\Psi>1$, so that voids may form by spontaneous nucleation. In addition, voids may form by homogeneous nucleation or by heterogeneous nucleation on clusters of $1,2,3$, or 4 helium atoms. The capture of 5 helium atoms gives a zero nucleation barrier. Figure 6 shows the progressive decrease in activation barrier with larger helium clusters acting as heterogeneous nucleation sites.

In the absence of helium, small vacancy clusters are free to decay to mono-vacancies. Such is not the case for clusters that contain helium. $\Delta G^{o}(j, x)$ in Eq. [14] is calculated assuming the van der Waals gas law, which ascribes a finite volume to each gas atom. Very small gas-containing cavities then have such a huge internal pressure that vacancy emission is impossible. This physical fact is reflected in the minima in the $\Delta G^{\prime} / \mathrm{kT}$ curves. Other gas laws give similar results.

Figure 7 shows nucleation rates calculated over the course of the $30 \mathrm{ks}$ irradiation for the same irradiation conditions as in Figures 5 and 6. Homogeneous nucleation dominates at all times, but at a decreasing rate. The decrease is caused by a lowered vacancy supersaturation because of an increasing void sink strength. Heterogeneous nucleation on clusters of 1 and 2 helium atoms begins early on but is soon eclipsed by nucleation

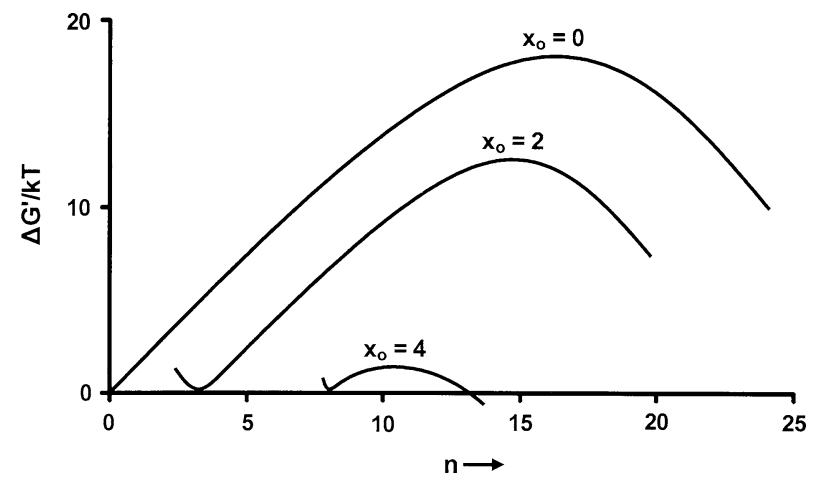

Fig. 6-Activation barriers for homogeneous nucleation and for heterogeneous nucleation on clusters of 2 and 4 helium atoms. ${ }^{[18]}$

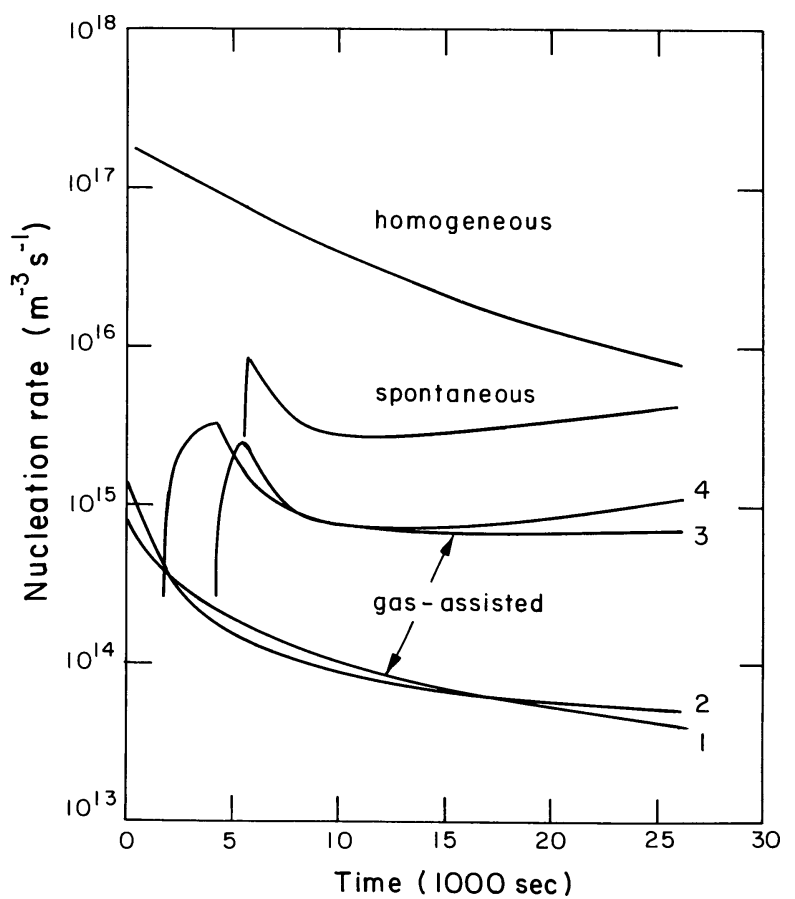

Fig. 7-Calculated void nucleation rates for the same conditions as Figs. 5 and 6. Homogeneous nucleation without gas is seen to dominate, though gas-assisted and spontaneous nucleation make significant contributions to the final void number density. ${ }^{[18]}$

on clusters of 3 and 4 helium atoms and by spontaneous nucleation. These latter three nucleation paths become operative only after the capture of the needed numbers of helium atoms.

Figure 8 shows calculated nucleation rates at a $100 \mathrm{~K}$ higher temperature and four times lower helium injection rate than in Figure 7. Again $\Psi>1$. However, the vacancy supersaturation is much reduced at the higher temperature, which leads to a much larger $\dot{n}$ nodal loop. Rates of homogeneous nucleation and heterogeneous nucleation on clusters of 1 or 2 helium atoms are greatly reduced because of the greater $\Delta G^{* \prime}$ s. Most nucleation is on clusters of 5 to 15 helium atoms. Spontaneous nucleation does not occur as capture of the needed 20 helium atoms is greater than $\tau_{r}$, which in this case is $30 \mathrm{ks}$.

At $773 \mathrm{~K}$, the vacancy supersaturation is extremely high, giving very rapid homogeneous nucleation. Helium-assisted nucleation of the sort illustrated in Figures 5 through 8 is so much slower that there is no significant contribution to the final number density of voids.

\section{VOID NUCLEATION SCENARIO WITH CLUSTER INJECTION}

First consider void nucleation with a linear rate of helium generation so that the $\dot{x}$ nodal line is initially coincident with the $n$-axis. Voids may form by homogeneous nucleation or by heterogeneous nucleation or on clusters injected from the displacement cascade. 


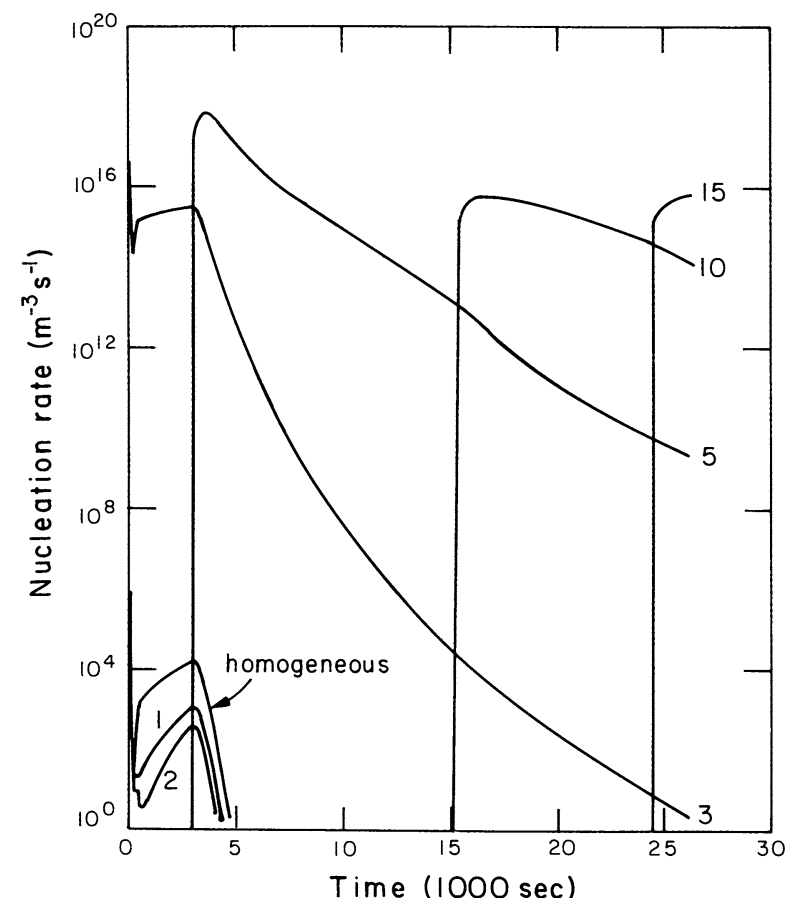

Fig. 8 - Calculated void nucleation rates at a $100 \mathrm{~K}$ higher temperature than in Fig. 7. Nucleation on larger clusters dominates, except at short times. ${ }^{[18]}$

The increasing helium concentration causes the $\dot{x}$ nodal line to rotate counterclockwise around the origin so that small helium clusters are stable. The injected clusters may capture helium if $\tau_{x}^{o}<\tau_{\delta}$. Capture of one or more helium atoms may take the cluster outside and to the right of the $\dot{n}$ nodal loop, where it is nucleated and able to grow. Otherwise the cluster will gain helium and lose vacancies until it reaches the $\dot{n}$ nodal line. This behavior is shown in Figure 3. In this way, the injected clusters may provide large embryos for heterogeneous nucleation even if cluster formation by single vacancy accretion is slow. Also, if $\Psi>0$, the clusters may move between the nodal lines to the top of the $\dot{n}$ nodal loop to give spontaneous nucleation.

Finally, the increasing void sink strength will so reduce the mobile helium concentration that the $\dot{x}$ nodal line will rotate clockwise, causing subcritical sized gas containing clusters to be unstable. Helium-assisted void nucleation will cease. Further irradiation will give void growth, but less nucleation. Thus, with or without cluster injection, helium-assisted void nucleation is likely to cease early in irradiations.

The scenario is somewhat different when a high concentration of helium is injected prior to the onset of displacement damage. Rapid void nucleation by the various mechanisms will begin immediately on onset of displacement damage. The injected clusters will capture helium to give nucleation if $\tau_{x}^{o}<\tau_{\delta}$. At the same time, $\Psi>1$ so that small helium-containing clusters readily form and contribute to the void nucleation rate as in the earlier case.

We recall here that the myriad of small vacancy clusters formed early in nucleation will not be deep traps for helium. Only voids of over 6 or 12 vacancies will constitute deep traps. It is the increasing number density of these deep traps that gives the precipitate drop in the concentration of mobile helium.

However, just what fraction of the injected clusters survives to become stable voids? Results of field ion microscopy and computer modeling studies indicate that a displacement cascade from a 2 to $30 \mathrm{keV}$ PKA is likely to produce hundreds of displaced atoms along with perhaps one cluster of 5 to 10 vacancies. We estimate the order of one large vacancy cluster per thousand displaced atoms. Irradiation to the relatively low dose of $1 \mathrm{dpa}$ then gives $\sim 10^{23}$ vacancies $/ \mathrm{cm}^{3}$ and $\sim 10^{20}$ large clusters $/ \mathrm{cm}^{3}$. Void number densities of are typically $\sim 10^{16} / \mathrm{cm}^{3}$, so survival of a very small fraction of these injected clusters would make a large contribution to the final void number density.

\section{CONCLUSIONS}

1. In the Poincaré critical point, a nodal line formalism has been used to analyze the synergistic effect of injected vacancy clusters and helium on void nucleation.

2. Helium: vacancy clusters have a diminished activation barrier to nucleation and are expected to play an important role in void nucleation under both neutron and charged-particle irradiations.

3. The detrapping of helium from voids is far more difficult than from dislocations or lattice sites. A modest number density of voids will trap the vast majority of the helium to stop helium-assisted void nucleation.

\section{FUTURE WORK}

The theory presented herein is suitable for numerical evaluation as part of a modeling study of void swelling. The results of such a study would be interesting.

Detrapping rates of helium from various traps are key quantities in determining the path of activation and void nucleation rate. Values used here are only rough estimates. Computer modeling could give much better values and make for a more accurate nucleation calculation.

Vacancy clusters formed in displacement cascades may play a key role in void nucleation. The modeling studies of Wirth[25] should be extended to other energy cascades and other matrix materials.

\section{NOMENCLATURE}

$A \quad$ energetic parameter in nucleus formation

$C_{i}, C_{v}, C_{x} \quad$ concentrations of self-interstitials, vacancies, and helium interstitials

$C_{o} \quad$ equilibrium vacancy concentration 
$D_{i}, D_{v}, D_{x}$

$J^{*}{ }^{s}$
$k_{c}^{2}$

$K_{i}, K_{v}$

$k_{i}^{2}, k_{v}^{2}, k_{x}^{2}$

$\mathrm{kT}$

$K_{x}^{c}$
$K_{x}^{T}$

$n$

$n^{*}$

$n_{o}$

$\hat{n}, \hat{x}$

$S_{e}$

$S_{v}$

$x$

Z

$Z_{i}, Z_{v}, Z_{x}$

$\alpha_{v}, \alpha_{x}$

$\beta^{*}$

$\beta_{i}, \beta_{v}, \beta_{x}$

$\beta_{i}^{o}, \beta_{v}^{o}, \beta_{x}^{o}$

$\gamma$

$\delta$

$\Delta G^{\prime}(n, x)$

$\Delta G^{*}$

$\Delta G^{o}(n, x)$

$\rho(x)$

$\rho_{c}$

$\rho_{d}$

$\tau_{i}^{o}, \tau_{v}^{o}, \tau_{x}^{o}$

$\tau_{j}$

$\tau_{r}$

$\tau_{v}, \tau_{i}, \tau_{x}$

$\tau_{\delta}$

$\Psi$

$\Omega$ concentration of trapped helium

diffusivities of self-interstitials, vacancies, and helium interstitials

steady-state nucleation rate

void sink strength for all defects

rates of self-interstitial and vacancy

production

dislocation sink strength for self-

interstitials, vacancies, or helium

Boltzmann factor

rate of helium detrapping from voids

rate of helium detrapping from vacancies

number of vacancies in a void

number of vacancies giving maximum

$\left(\Delta G^{*}\right)$ in $\Delta G^{\prime}\left(n, x_{o}\right)$

number of vacancies giving minimum in

$\Delta G^{\prime}\left(n, x_{o}\right)$

coordinates of stable node in Poincaré

analysis

radiation modified vacancy

supersaturation

vacancy supersaturation

number of helium atoms in a void

Zeldovich factor

dislocation sink strength constants for self-interstitials, vacancies, and helium interstitials

emission rates of vacancies and helium

atoms from voids

impingement rate of vacancies on the critical nucleus

impingement rates of self-interstitials, vacancies, and helium on a void impingement rates of self-interstitials, vacancies, and helium on a mono-vacancy void: matrix surface energy

width of $\Delta G^{\prime}(n, x), \mathrm{kT}$ below its maximum radiation-modified barrier for void nucleation

value of $\Delta G^{\prime}(n, x)$, at its maximum

thermodynamic barrier to void nucleation number density of clusters of $x$ gas atoms total number density of voids

dislocation number density

Reciprocals of $\beta_{i}^{o}, \beta_{v}^{o}, \beta_{x}^{o}$

time to largely complete nucleation.

duration of radiation

times between arrivals of vacancies, selfinterstitials, or helium atoms at a void

incubation time for steady-state

nucleation

constant governing nodal lines

atomic volume of solid

\section{REFERENCES}

1. C. Cawthorne and E.J. Fulton: Nature, 1966, vol. 216, pp. 575-76.

2. Radiation-Induced Voids in Metals, J.W. Corbett and L.C. Ianniello, eds., USAEC Symp. Series 26, Conf-710601, 1972.

3. K.C. Russell: Acta Metall., 1971, vol. 19, pp. 753-58.

4. J.L. Katz and H. Wiedersich: J. Chem. Phys., 1971, vol. 55, pp. 1414-25.

5. W.D. Wilson and R.A. Johnson: in Interatomic Potentials and Simulation of Point Defects, P.C. Gehlen, J.R. Beeler, Jr., and R.I. Jafee, eds., Plenum Press, New York, NY, 1972, p. 375.

6. J.L. Katz and H. Wiedersich: J. Nucl. Mater., 1973, vol. 46, pp. 41-45.

7. H. Wiedersich, J.J. Burton, and J.L. Katz: J. Nucl. Mater., 1974, vol. 51, pp. 287-301.

8. H. Wiedersich and B.O. Hall: J. Nucl. Mater., 1977, vol. 56, pp. 187-92.

9. H. Wiedersich and J.L. Katz: Adv. Colloid Interface Sci., 1979, vol. 10 , pp. 33-71.

10. K.C. Russell: Acta Metall., 1972, vol. 20, pp. 899-907.

11. K.C. Russell: Scripta Metall., 1972, vol. 6, pp. 209-14.

12. K.C. Russell and D.H. Hall: in Defects and Defect Clusters in BCC Metals and Their Alloys, R. Arsenault, ed., National Bureau of Standards, Washington, DC, 1973, pp. 545-64.

13. K.C. Russell: Scripta Metall., 1973, vol. 7, pp. 755-60.

14. K.C. Russell: in The Physics of Irradiation Produced Voids, R.S. Nelson, ed., HMSO, London, 1975, pp. 158-79.

15. K.C. Russell: Acta Metall., 1978, vol. 26, pp. 1615-30.

16. C.A. Parker and K.C. Russell: Scripta Metall., 1981, vol. 15, pp. 643-47.

17. C.A. Parker and K.C. Russell: in Effects of Radiation on Materials, ASTM STP 782, H.R. Brager and J.S. Perrin, eds., ASTM, Philadelphia, PA, 1982, pp. 1042-53.

18. C.A. Parker and K.C. Russell: J. Nucl. Mater., 1983, vol. 119, pp. 82-94.

19. A.F. Calder and D.J. Bacon: J. Nucl. Mater., 1993, vol. 207, pp. $25-45$.

20. R.E. Stoller: J. Nucl. Mater., 2004, vol. 276, pp. 22-32.

21. T.D. de la Rubia and M.W. Guinan: Mater. Sci. Forum, 1992, vols. $97-98$, pp. $23-42$.

22. D.J. Bacon and T.D. de la Rubia: J. Nucl. Mater., 1994, vol. 216, pp. $275-90$.

23. C.H. Woo and B.N. Singh: Philos. Mag. A, 1992, vol. 65, p. 889.

24. C.-Y. Wei, M.I. Current, and D.N. Seidman: Philos. Mag. A, 1981, vol. 44, pp. 459-91.

25. B. Wirth: Ph.D. Thesis, University of California Santa Barbara, Santa Barbara, CA, 1998.

26. M.J. Caturla, T.D. de la Rubia, and M. Fluss: J. Nucl. Mater., 2003, vol. 323, pp. 163-68.

27. M.J. Caturla, N. Soneda, E. Alonso, B.D. Wirth, T.D. de la Rubia, and J.M. Perlado: J. Nucl. Mater., 2000, vol. 276, pp. 13-21.

28. K.C. Russell and B.K. Kim: J. Nucl. Mater., 2006, vol. 351, pp. $100-8$.

29. P. Erhart, W.G. Hanbold, and W. Schilling: Festkörperprobleme, 1974, vol. 14, p. 4187.

30. H. Wiedersich: Radiat. Eff. Def. Solids, 1972, vol. 12, p. 1111.

31. A.D. Brailsford and R. Bullough: J. Nucl. Mater., 1972, vol. 44, p. 121.

32. H. Poincaré: Les Methodes de la Mecanique Celeste, GauthiersVillars, Paris, 1892-1899, vols. I-III.

33. J. Feder, K.C. Russell, J. Lothe, and G.M. Pound: Adv. Phys., 1966, vol. 15, pp. 111-78. 